\title{
Inhibitory effect of hyperoside isolated from Zanthoxylum bungeanum leaves on SW620 human colorectal cancer cells via induction of the p53 signaling pathway and apoptosis
}

\author{
YALI ZHANG ${ }^{1}$, HUANHUAN DONG $^{1}$, JINGFANG ZHANG ${ }^{2}$ and LIYU ZHANG $^{3}$ \\ ${ }^{1}$ Key Laboratory of Biomedical Information Engineering of Ministry of Education, School of Life Science \\ and Technology, Xi'an Jiaotong University, Xi'an, Shaanxi 710049; ${ }^{2}$ College of Forestry, \\ Northwest A\&F University, Xianyang, Shaanxi 712100; ${ }^{3}$ Shaanxi Institute of Pediatric Diseases, \\ Xi'an Children's Hospital, Xi'an, Shaanxi 710002, P.R. China
}

Received May 20,2016; Accepted April 6, 2017

DOI: $10.3892 / \mathrm{mmr} .2017 .6710$

\begin{abstract}
The present study aimed to demonstrate the antiproliferative effect of hyperoside from Zanthoxylum bungeanum leaves (HZL) and explain the underlying molecular mechanisms in the SW620 human colorectal cancer cell line. The cytotoxic effects of HZL were determined using a3-(4,5-dimethylthiazol-2-yl)2,5-diphenyltetrazolium bromide assay. Apoptosis and cell cycle were detected using flow cytometry. Reactive oxygen species (ROS) levels and mitochondrial membrane potential $\left(\Delta \Psi_{\mathrm{m}}\right)$ were assessed using 2',7'-dichlorofluorescin diacetate and 5,5',6,6'-tetrachloro-1,1',3,3'-tetraethylbenzimidazolyl carbocyanine iodide fluorescence spectrophotometry, respectively. Western blot analysis was used to quantify the expression levels of apoptosis-associated proteins. Reverse transcription-quantitative polymerase chain reaction analysis was used to determine the mRNA expression of glutathione peroxidase (GSH-Px) and catalase (CAT). HZL had a marked anti-proliferative effect on the SW620 human colorectal cancer cells by inducing cell cycle G2/M phase arrest and apoptosis, which was associated with an increase in the expression of p53 and p21. Further mechanistic investigations revealed that the induction of apoptosis was associated with increased generation of ROS, reduced $\Delta \Psi_{\mathrm{m}}$, and upregulation of B-cell lymphoma 2-associated X protein, cytochrome $c$, caspase-9, apoptotic protease activating factor 1 and caspase-3. The antitumor potency of HZL was also attributed to inhibition of the mRNA expression levels of GSH-Px and CAT. These
\end{abstract}

Correspondence to: Professor Jingfang Zhang, College of Forestry, Northwest A\&F University, 3 Taicheng Road, Yangling, Xianyang, Shaanxi 712100, P.R. China

E-mail: zjf19890117@163.com

Key words: hyperoside, Zanthoxylum bungeanum, apoptosis, p53, reactive oxygen species, SW620 human colorectal cancer cells data indicated that HZL may be involved in the pro-apoptotic signaling of SW620 human colorectal cancer cells via induction of the caspase-dependent apoptosis and p53 signaling pathways.

\section{Introduction}

Colorectal carcinoma (CRC) is one of the most common types of human malignancy and a leading cause of cancer-associated mortality in developed countries (1). Identifying strategies to fight against CRC is important, as it is an emerging health problem. It is now well documented that phytochemicals, including flavonoids, alkaloids and polysaccharides, are important in anticancer therapy $(2,3)$.

Zanthoxylum bungeanum, also known as Sichuan pepper, is a common Chinese culinary herb, growing widely in the Sichuan, Shaanxi, Shandong and Hebei provinces of China. $Z$. bungeanum leaves are generally used as a vegetable (4). Previous studies have reported on $Z$. bungeanum leaves, in which its antioxidant (5-7), antimicrobial (8), antithrombotic (9) and lipid metabolism-regulating effects (10) were evaluated. However, there has been limited focus on the anticancer activity of the components of $Z$. bungeanum leaves. Hyperoside (quercetin-3-O-galactoside; Fig. 1) is one of the primary flavonoid compounds in $Z$. bungeanum leaves. In addition to its potent efficacy for pain relief, anti-inflammatory and antioxidant activity, and its potential for protecting the cardiovascular system (11-13), hyperoside shows antitumor activity in several tumor models, including RL952 endometrial carcinoma cells (14), A549 lung adenocarcinoma cells $(15,16), 786-O$ renal cancer cells (17) and PC3 prostate cancer cells (12). However, the molecular mechanisms underlying hyperoside-induced growth inhibition and apoptosis of CRC cells remain to be fully elucidated.

A number of flavonoids have been reported to exert pro-oxidant actions, which may be an important mechanism underlying their anticancer and apoptosis-inducing properties (18). It is well documented that flavonoids generate reactive 
oxygen species (ROS) in cancer cells. Enhanced ROS production leads to the disruption of cellular antioxidant defense systems and the release of cytochrome $c$ from the mitochondria to the cytosol, resulting in apoptotic cell death (19). Tumor cells with higher levels of ROS are more susceptible to cell death, compared with normal cells with lower levels of ROS. Therefore, novel anticancer drugs may have high potential in promoting the production of ROS. Accumulating evidence suggests that the tumor suppressor, p53, is central to the process of ROS-mediated apoptosis (20). In the present study, hyperoside was isolated from Z. bungeanum leaves (HZL) and its effect on caspase-dependent apoptosis and p53 signaling pathways in human SW620 CRC cells was investigated. The results demonstrated that hyperoside induced cell cycle G2/M phase arrest and apoptosis, which was associated with an increase in the levels of p53, p21, B-cell lymphoma 2 (Bcl-2)-associated $\mathrm{X}$ protein (Bax), cytochrome $c$, caspase- 9 , caspase-3 and apoptotic protease activating factor 1 (Apaf-1). The antitumor potency of HZL was also attributed to increasing ROS levels, a reduction in mitochondrial membrane potential $\left(\Delta \Psi_{\mathrm{m}}\right)$, and downregulation of the mRNA expression of glutathione peroxidase (GSH-Px) and catalase (CAT).

\section{Materials and methods}

Materials. The leaves of $Z$. bungeanum were harvested in Shaanxi, China in August 2015 and were identified according to the identification standards of the Pharmacopeia of the People's Republic of China (21). Dulbecco's modified Eagle's medium (DMEM), dimethyl sulfoxide (DMSO), 3-(4,5-dimethylthiazol-2-yl) 2,5-diphenyltetrazolium bromide (MTT) and 2',7'-dichlorofluorescin diacetate (DCFH-DA) were obtained from Sigma-Aldrich; Merck KGaA (Darmstadt, Germany). Fetal bovine serum (FBS) was purchased from Gibco; Thermo Fisher Scientific, Inc. (Waltham, MA, USA). 5,5',6,6'-tetrachloro-1,1',3,3'-tetraethylbenzimidazolyl carbocyanine iodide (JC-1) was purchased from Beyotime Institute of Biotechnology (Shanghai, China). The primers for GSH-Px and CAT were designed and synthesized by Takara Biotechnology Co., Ltd. (Dalian, China). Reagents, including enzymes, cofactors and nucleotides for internal standard construction and reverse transcription-quantitative polymerase chain reaction (RT-qPCR) analysis, were obtained from Takara Biotechnology Co., Ltd.

Preparation of HZL.Z. bungeanum leaves $(500 \mathrm{~g})$ were soaked in $70 \%$ ethanol $(1: 10, \mathrm{w} / \mathrm{v})$ for $2.5 \mathrm{~h}$ and were sonicated in an ultrasonic bath at $200 \mathrm{kHz}$ at $55^{\circ} \mathrm{C}$ for $45 \mathrm{~min}$. The samples were filtered, concentrated and then dried using a rotary evaporator. The dried crude extract was added to distilled water and defatted with petroleum ether. The residue was diluted in $\mathrm{H}_{2} \mathrm{O}$ and extracted using ethyl acetate in triplicate. The resulting fractions were chromatographed over acetonitrile- $0.2 \%$ acetic acid $(15: 85, \mathrm{v} / \mathrm{v})$. The chemical identity of hyperoside was confirmed using reverse phase high performance liquid chromatography by comparison with authentic hyperoside (National Institute for the Control of Pharmaceutical and Biological Products, Beijing, China).

The hyperoside was dissolved in DMSO immediately prior to use, and the final concentration of DMSO was $<0.1 \%$ (v/v)

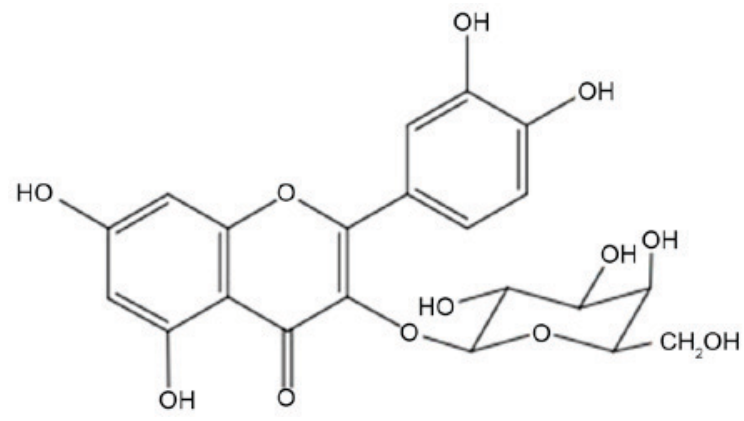

Figure 1. Structure of hyperoside (quercetin-3-O-galactoside).

in all experiments. The concentrations of hyperoside ranged between 12.5 and $50 \mu \mathrm{M}$. DMSO at $0.1 \%$ was used as a control. All determinations were performed in triplicate.

Cell culture and treatment. SW620 cells from the American Type Culture Collection (ATCC; Manassas, VA, USA) were maintained in DMEM supplemented with $10 \%$ heat-inactivated $\mathrm{FBS}, 100 \mathrm{U} / \mathrm{ml}$ penicillin and $100 \mu \mathrm{g} / \mathrm{ml}$ streptomycin (Thermo Fisher Scientific, Inc.) in a humidified $5 \% \mathrm{CO}_{2}$ incubator at $37^{\circ} \mathrm{C}$. The medium was replaced every 2 days. The SW620 cells were cultured in 24- or 96-well plates at a density of $1-2 \times 10^{5}$ cells $/ \mathrm{ml}$ and were grown to $\sim 70 \%$ confluence.

Cell viability. The cell survival rate was quantified using a colorimetric MTT assay. MTT was prepared at $2.5 \mathrm{mg} / \mathrm{ml}$ in phosphate-buffered saline (PBS). Briefly, $20 \mu 1$ aliquots of the MTT stock solution were pipetted into each well, and the plates were incubated at $37^{\circ} \mathrm{C}$ in a humidified $5 \% \mathrm{CO}_{2}$ incubator. After $4 \mathrm{~h}$, the medium was removed and DMSO $(200 \mu \mathrm{l})$ was added to each well to dissolve the formazan. The optical density of each well was measured 10 min later at $570 \mathrm{~nm}$ using a spectrophotometer (Tecan Infinite M200 Pro; Tecan Group Ltd., Männedorf, Switzerland).

Flow cytometric analysis for cell cycle phase determination. Cell suspensions $\left(0.5-1 \times 10^{5} / \mathrm{ml}\right)$ were prepared by trypsinization and washed twice with PBS, followed by centrifugation $(200 \mathrm{x} \mathrm{g})$ for $5 \mathrm{~min}$ at $4^{\circ} \mathrm{C}$. The cells were fixed with $70 \%$ ethanol at $4^{\circ} \mathrm{C}$ and resuspended in PBS, which contained $0.25 \mathrm{mg} / \mathrm{ml}$ of RNaseA (Thermo Fisher Scientific, Inc.). The suspension was incubated for $30 \mathrm{~min}$ at $37^{\circ} \mathrm{C}$, following which the cells were labeled with $50 \mu \mathrm{g} / \mathrm{ml}$ propidium iodide (PI; Sigma-Aldrich; Merck KGaA). The total DNA content was quantified by fluorescence using a FACS flow cytometer (BD Biosciences, Franklin Lakes, NJ, USA).

Flow cytometric analysis of apoptosis. The prepared SW620 cells $\left(1 \times 10^{6} / \mathrm{ml}\right)$ were washed twice with ice-cold PBS and gently resuspended in $500 \mu \mathrm{l}$ of binding buffer. Subsequently, the cells were stained with $5 \mu \mathrm{l}$ Annexin V-FITC and agitated well. Finally, $5 \mu 1$ PI was added to the cells, incubated for $20 \mathrm{~min}$ in the dark and then analyzed using a FACS flow cytometer (BD Biosciences).

Western blot analysis. Cell lysates were prepared from confluent 6-well plates (Nalge Nunc International, Penfield, 
NY, USA) by scraping into lysis buffer [0.05 M Tris- $\mathrm{HCl}$, $10 \%(\mathrm{v} / \mathrm{v})$ glycerol, $2 \%(\mathrm{w} / \mathrm{v}) \mathrm{SDS}]$. The total protein content of cell lysates was determined by the Bradford method (Bio-Rad Laboratories, Inc., Hercules, CA, USA). Cell lysates (30 $\mu \mathrm{g}$ of total protein) were analyzed using $8-12 \%$ gradient SDS-PAGE. The proteins were transferred onto an immunoblot PVDF membrane (EMD Millipore, Billerica, MA, USA), which was blocked with $5 \%$ skimmed milk in TBS containing $0.1 \%$ Tween-20 (TBS-T) at room temperature for $1 \mathrm{~h}$, and incubated overnight at $4^{\circ} \mathrm{C}$ with the following primary antibodies: Rabbit anti-p53 (4667; 1:1,000), anti-p21 (2947; 1:2,000), anti-Bax (2772s; 1:1,000), anticytochrome $c(4280 \mathrm{~s} ; 1: 1,000)$, anti-Apaf-1 $(8969 \mathrm{~s} ; 1: 1,000)$, anti-caspase-9 (9508; 1:1,000), or anti-caspase-3 (9662s; 1:1,000) (all from Cell Signaling Technology, Inc., Danvers, MA, USA). The membranes were then washed with TBS-T buffer, incubated with the horseradish peroxidase (HRP)conjugated anti-mouse IgG (14709; 1:1,000; Cell Signaling Technology, Inc.) for $1 \mathrm{~h}$ at room temperature, and enhanced using a chemiluminescence ECL assay kit (Amersham; GE Healthcare Life Sciences, Chalfont, UK) according to the manufacturer's protocol. Images of chemiluminescence were captured using a Fujifilm LAS-3000 system (Fujifilm, Tokyo, Japan). The basal levels of proteins were normalized to the protein level of $\beta$-actin.

Measurement of $\Delta \Psi m$. The $\Delta \Psi_{\mathrm{m}}$ of the cells was analyzed using fluorescence spectrophotometry using JC-1 dye. Briefly, the SW620 cells were stained with $5 \mathrm{mM} \mathrm{JC}-1$ at $37^{\circ} \mathrm{C}$ for $20 \mathrm{~min}$ in a $5 \% \mathrm{CO}_{2}$ atmosphere. The cells were pelleted by centrifugation $\left(1,000 \mathrm{x}\right.$ g for $5 \mathrm{~min}$ at $\left.4^{\circ} \mathrm{C}\right)$ and resuspended in PBS. The JC-1 fluorescence of the cell suspensions and PBS controls were measured in triplicate in Costar 96-well plates using a microplate reader (Tecan Infinite M200 Pro; Tecan Group Ltd.). Ex/Em (green)/Em (red) $=485 / 538 / 590 \mathrm{~nm}$; FL2/FL1 ratios were calculated based on the fluorescence data. Each well was scanned by measuring the intensity of each 25 -square grid $\left(1 \mathrm{~mm}^{2}\right.$ area), which was arranged in a $5 \times 5$ rectangular array (bottom scanning). A higher red-to-green ratio indicated a more polarized or more negative and hyperpolarized mitochondrial inner membrane.

Measurement of intracellular ROS levels. The cellular ROS levels were measured using a dichlorofluorescein assay. The cells were collected and incubated with $100 \mu \mathrm{M}$ DCF-DA (dissolved in DMSO) for $30 \mathrm{~min}$ at $37^{\circ} \mathrm{C}$. Subsequently, the cells were washed three times with PBS ( $\mathrm{pH} \mathrm{7.4)} \mathrm{and} \mathrm{the}$ relative levels of fluorescence were quantified in a spectrophotofluorimeter (Tecan Infinite M200 Pro; Tecan Group, Ltd.). The measured fluorescence values were expressed as a percentage of the fluorescence in the control cells.

RT-qPCR analysis. The cells were washed with ice-cold PBS, and RNA was extracted using TRIzol reagent (Bioteke Corporation, Beijing, China) according to the manufacturer's protocol. cDNA was synthesized from mRNA using a PrimeScript ${ }^{\mathrm{TM}}$ RT reagent kit (Takara Biotechnology Co., Ltd.), followed by qPCR using a SYBR Premix Ex Taq ${ }^{\mathrm{TM}}$ reagent kit (Takara Biotechnology Co., Ltd.) and the ABI
Prism 7500 sequence detection system (Applied Biosystems; Thermo Fisher Scientific, Inc.). The reactions were performed under the following cycling conditions: $95^{\circ} \mathrm{C}$ for $30 \mathrm{sec}$, followed by 40 cycles of $95^{\circ} \mathrm{C}$ for $5 \mathrm{sec}$ and $56^{\circ} \mathrm{C}$ for $30 \mathrm{sec}$. The housekeeping gene $\beta$-actin was used for normalization. All experiments were repeated at least three times. The relative changes in gene expression were analyzed using the $2^{-\Delta \Delta \mathrm{Cq}}$ method (22). The sequences for the RT-qPCR primers were as follows: GSH-Px forward, 5'-CCTCTAAACCTACGAGGG AGGAA-3' and reverse, 5'-GGGAAACTCGCCTTGGTCT-3'; CAT forward, 5'-TCCAAGGCAAAGGTATTTGAGCA-3' and reverse, 5'-CAACGAGATCCCAGTTACCATCTTC-3'; $\beta$-actin forward, 5'-CATCCGTAAAGACCTCTATGCCAA C-3' and reverse, 5'-ATGGAGCCACCGATCCACA-3'.

Statistical analysis. All data are expressed as the mean \pm standard deviation of at least three independent determinations for each experiment. Statistical analyses were performed using SPSS version 13.0 (SPSS, Inc., Chicago, IL, USA). One-way analysis of variance with Duncan's multiple range test was used to examine differences between groups. $\mathrm{P}<0.05$ was considered to indicate a statistically significant difference.

\section{Results}

Effects of HZL on SW620 cell growth inhibition. The growth inhibiting effect of HZL was assessed using an MTT assay and microscopic analysis, as demonstrated in Fig. 2A. Timeand concentration-dependent growth inhibition was observed in the SW620 human CRC cell line. Following treatment with HZL for 24, 48 and $96 \mathrm{~h}$, the half maximal inhibitory concentration values were $72.35 \pm 5.46,36.41 \pm 1.36$ and $19.51 \pm 4.95 \mu \mathrm{M}$, respectively. The viabilities of cells treated with HZL $(12.5,25$ and $50 \mu \mathrm{M})$ for $48 \mathrm{~h}$ were significantly $(\mathrm{P}<0.01, \mathrm{P}<0.01$ and $\mathrm{P}<0.01$, respectively) lower, compared with cells in the control group (Fig. 2A). Additionally, microscopical analysis demonstrated prominent morphological changes resembling cell swelling, irregularities in the plasma membrane and the formation of membrane blebs and vacuoles, to a significantly higher extent, compared with the control cells (Fig. 2B). This provided morphological evidence to qualitatively demonstrate that HZL induced apoptosis, which was in agreement with the results of the MTT assay described above.

The present study then investigated whether the cytotoxicity of HZL to SW620 cells is associated with the induction of cell cycle arrest and apoptosis. Cell cycle arrest and apoptosis were determined using flow cytometry following treatment of the SW620 cells with 12.5, 25 and $50 \mu \mathrm{M}$ HZL for $48 \mathrm{~h}$. Characteristic examples of the observations are demonstrated in Fig. 2C and D. The percentages of SW620 cell apoptosis were 17.3, 21.2 and 32.9\%, in the12.5, 25 and $50 \mu \mathrm{M}$ HZL treatment groups, respectively (Fig. 2C). These results demonstrated that HZL induced apoptosis in a dose-dependent manner, which was consistent with the results of the MTT assay. The percentage of $\mathrm{G} 2 / \mathrm{M}$ phase cells in the groups treated with 12.5, 25 and $50 \mu \mathrm{M}$ HZLwas significantly elevated, whereas the percentages of $\mathrm{G} 1 / \mathrm{G} 0$ and $\mathrm{S}$ phase cells were decreased by HZL (Fig. 2D). 
A

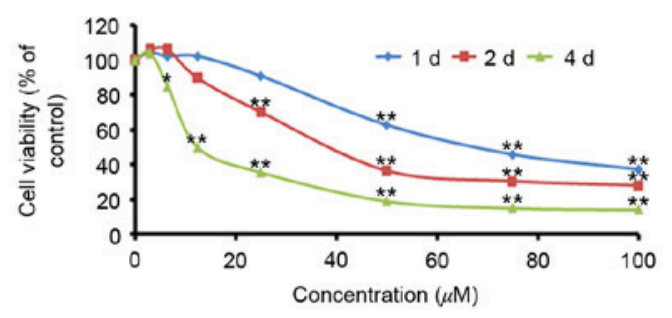

c
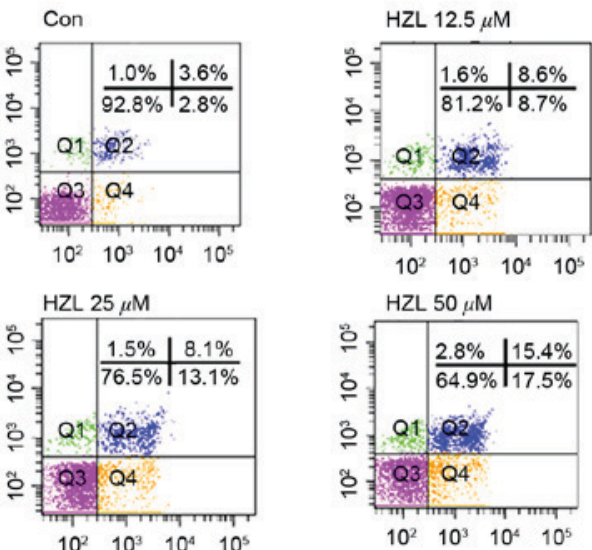

$\mathrm{HZL} 50 \mu \mathrm{M}$

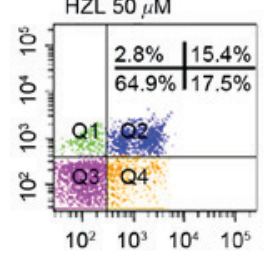

B

D

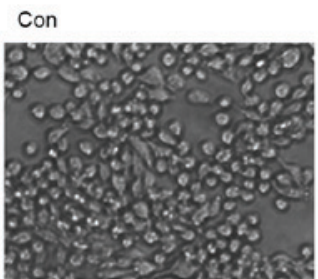

HZL $25 \mu \mathrm{M}$

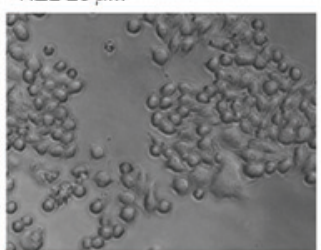

HZL $12.5 u \mathrm{M}$

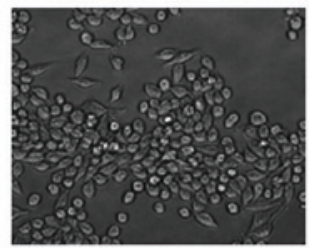

HZL $50 \mu \mathrm{M}$

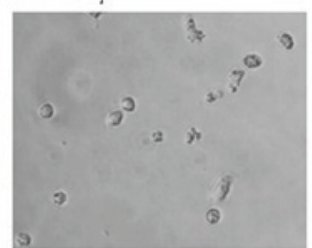

$\mathrm{HZL} 12.5 \mu \mathrm{M}$
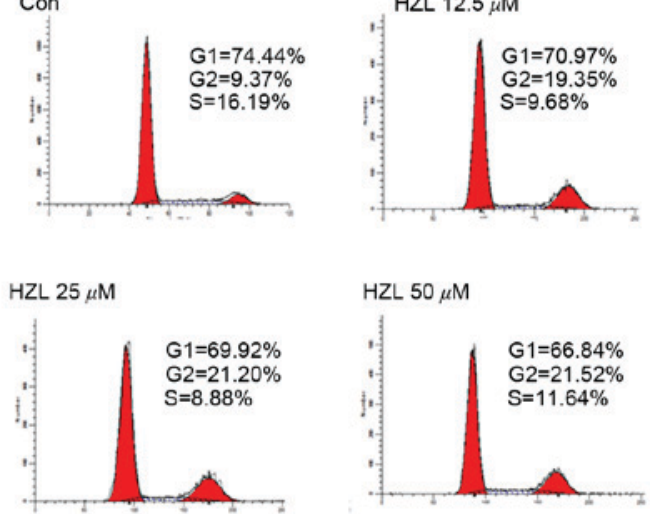

Figure 2. Inhibitory effect of HZL on the proliferation of SW620 cells. (A) Cell viability. (B) Morphological changes (magnification, x200). (C) Assessment of apoptosis. (D) Cell-cycle analysis. ${ }^{*} \mathrm{P}<0.05$ and ${ }^{* *} \mathrm{P}<0.01$ vs. control. Con, control; HZL, Zanthoxylum bungeanum.

p53-mediated apoptosis. In order to examine the mechanisms underlying HZL-induced cell cycle arrest and apoptosis, activation of the p53-mediated signaling network in SW620 cells was investigated using western blot analysis (Fig. 3A). Compared with the control group, the protein expression levels of p53 and p21 were increased by 116 and $198 \%$, respectively, in the SW620 cells treated with $25 \mu \mathrm{M}$ HZL for $48 \mathrm{~h}$ (Fig. 3B and C). The expression levels of pro-apoptotic proteins Bax, cytochrome $c$, Apaf-1, caspase- 9 and caspase-3 were increased in a dose-dependent manner (Fig. 3D-H). These data suggested that HZL induced apoptosis of the SW620 cells through p53- and caspase-dependent apoptotic pathways.

ROS-mediated apoptosis. The enhancement of ROS production has long been associated with the apoptotic response induced by anticancer agents. Mitochondria are key organelles for cell survival and area source of ROS generation during apoptosis. In the present study, JC-1 staining was applied to detect changes in the $\Delta \Psi_{\mathrm{m}}$ of SW620 cells. The ratios of JC-1 red-to-green fluorescence intensities decreased by $21.8,41.6$ and $43.7 \%$ when the SW620 cells were treated with 12.5, 25 and $50 \mu \mathrm{M}$ HZL for $48 \mathrm{~h}$. These results revealed that HZL induced apoptosis through the mitochondrial pathway (Fig. 4A). The generation of ROS was increased to $139.1 \%$ of the untreated control when treated with $25 \mu \mathrm{M}$ HZL for $48 \mathrm{~h}$ (Fig. 4B). This indicated that HZL caused the production of ROS. ROS are created through aerobic metabolism and are rapidly removed by endogenous antioxidants, including superoxide dismutase
(SOD), CAT and GSH-Px. The results of the present study demonstrated that the mRNA expression levels of CAT and GSH-Px were decreased following treatment with $25 \mu \mathrm{M}$ HZL, whereas the ROS levels were increased (Fig. 4C and D).

\section{Discussion}

Flavonoids are polyphenolic compounds, which are found ubiquitously in plants. The role of dietary flavonoids in cancer prevention has been widely discussed. Several mechanisms of action of flavonoids have been identified, including carcinogen inactivation, antiproliferative activity, cell cycle arrest, induction of differentiation and apoptosis, inhibition of angiogenesis, antioxidant activity and reversal of multidrug resistance, or a combination of these mechanisms (23). Hyperoside is one of the major active compounds of $Z$. bungeanum leaves; however, the anticancer activity of hyperoside has not been reported previously. To the best of the authors' knowledge, the present study is the first to investigate the molecular effects of HZL on human SW620 CRC cells.

Inhibition of the cell cycle has become an important target for the management of cancer. In the present study, the data obtained on the cell cycle distribution of the cell population indicated that HZL may have mediated the growth inhibition of SW620 cells via perturbation in the G2/M phase of the cell cycle. It has been demonstrated that flavonoids induce the inhibition of growth via cell cycle arrest in the G2/M phase of several cancer cell lines, including ovarian carcinoma 
A

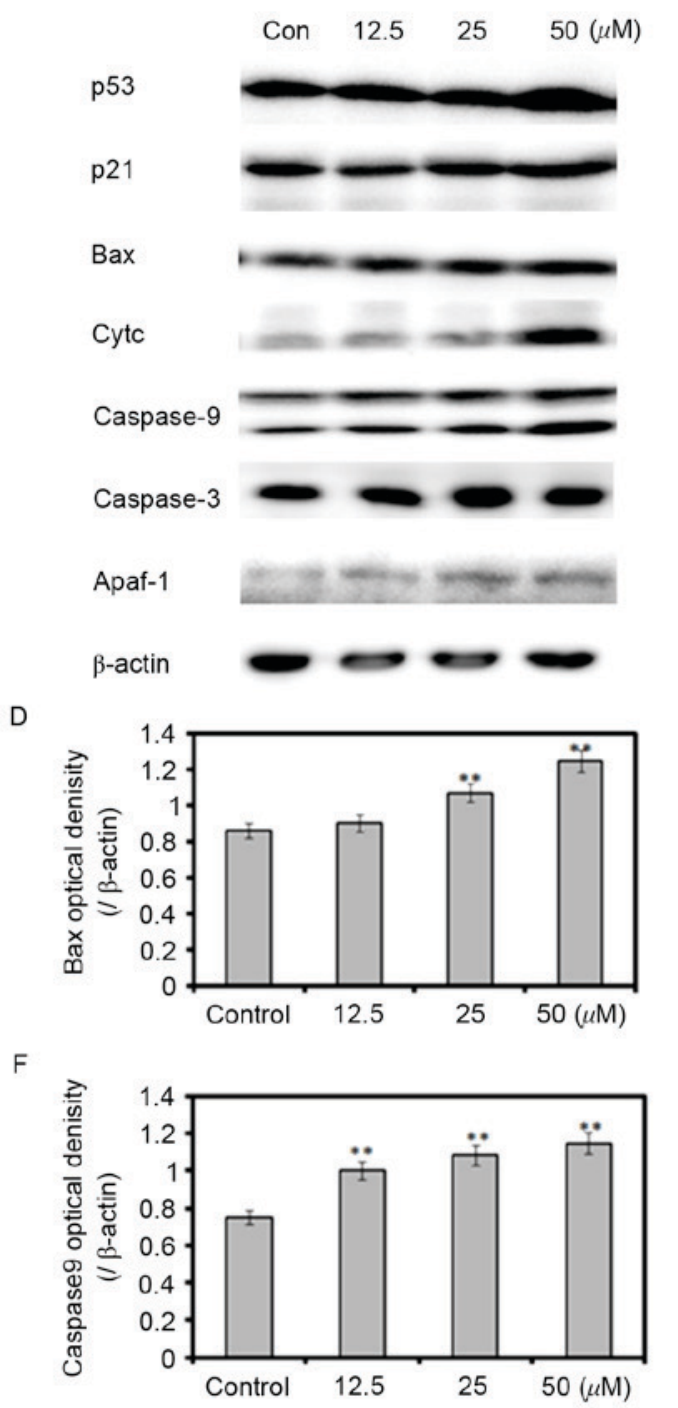

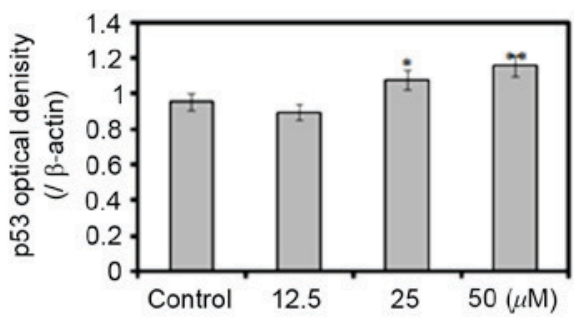

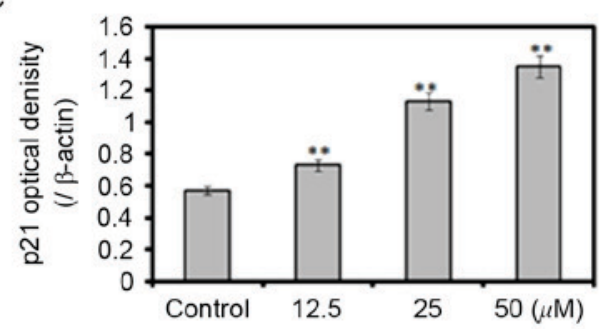

$E$
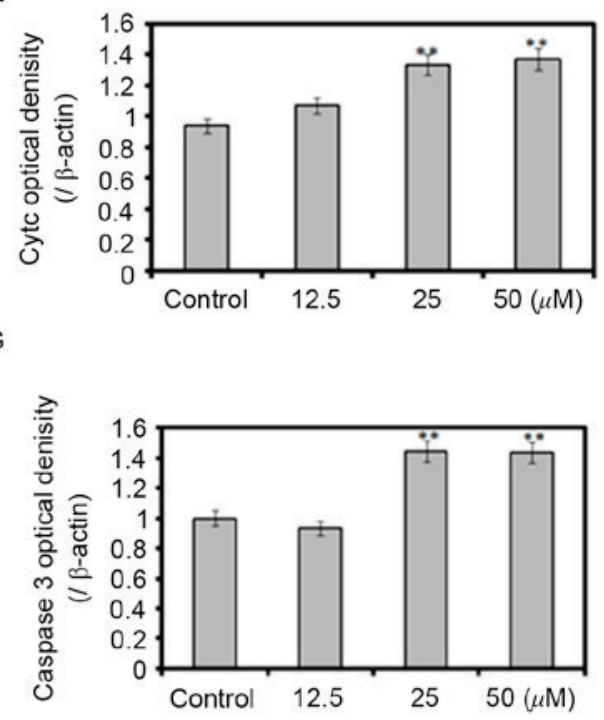

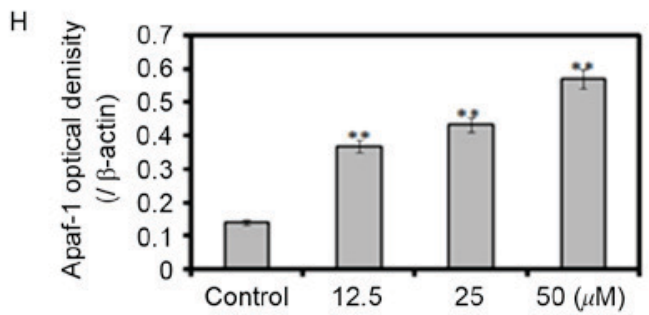

Figure 3. Effect of Zanthoxylum bungeanum on p53-associated proteins. SW620 cells were exposed to $12.5,25$ and $50 \mu \mathrm{M}$ hyperoside for 48 h. (A) Protein expression of p53-associated proteins. Quantification of (B) p53, (C) p21, (D) Bax, (E) Cytc, (F) caspase-9, (G) caspase-3 and (H) Apaf-1. ${ }^{*}<0.05$ and ${ }^{* *} \mathrm{P}<0.01$ vs. Control. Bax, Bcl-2-associated X protein; Cytc, cytochrome c; Apaf-1, apoptotic protease activating factor 1.

(SKOV3) and osteosarcoma (U2OS) (24), anaplastic thyroid carcinoma (25), and several digestive system cancer cell lines $(26,27)$, which in itself is inconsistent with the results obtained in the present study. However, Zhang et al (28) investigated the effect of hyperoside on human osteosarcoma cells and found that, following treatment with hyperoside, cells were arrested in the G0/G1 phase. Several studies have also indicated that arrest of the cell cycle in the G0/G1 phase is induced by flavonoids in human osteosarcoma MG-63, HeLa, prostate and breast cancer cells (29-31). Therefore, the cellular changes referred to above suggest that the cell cycle arrest caused by flavonoids is cell type- and concentration-dependent.

Tumor suppressor p53 is important in mediating cell responses to various stresses, predominantly by inducing or suppressing a number of genes involved in cell processes, including cell death, cell cycle arrest, apoptosis, senescence and DNA-repair $(32,33)$. p21 is an important checkpoint gene in the cell cycle, and it is also regulated by the transcription of p53; it contributes to the repair of damaged cells by terminating DNA synthesis and inactivating the nuclear antigen in proliferating cells (34). As demonstrated in Fig. 3, the present 
A

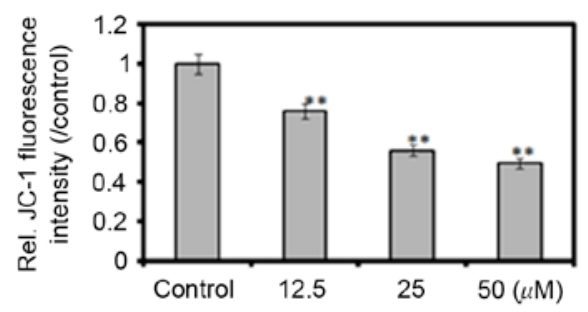

C

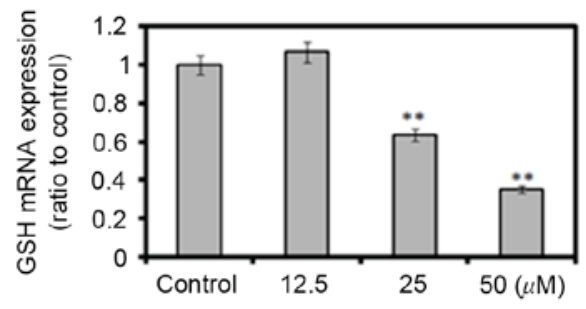

B

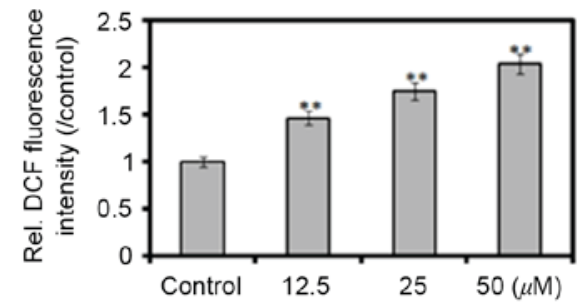

D

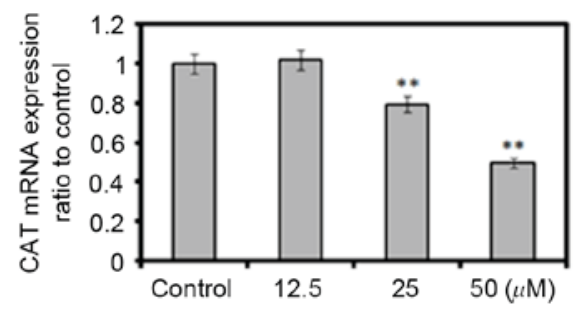

Figure 4. Effect of Zanthoxylum bungeanum on $\Delta \Psi \mathrm{m}$, ROS and the gene expression of antioxidant enzymes. Cells were treated with $12.5,25$ and $50 \mu \mathrm{M}$ hyperoside for $48 \mathrm{~h}$. (A) $\Delta \Psi_{\mathrm{m}}$ measured using a JC-1 assay; (B) Reactive oxygen species production determined using the DCFH-Da probe; mRNA expression of (C) GSH-Px and (D) CAT determined using reverse transcription-quantitative polymerase chain reaction analysis. ${ }^{* *} \mathrm{P}<0.01$ vs. control. GSH-Px, glutathione peroxidase; CAT, catalase; DCFH-Da, 2',7'-dichlorofluorescin diacetate; Rel., relative; JC-1, 5,5',6,6'-tetrachloro-1,1',3,3'-tetraethylbenzimidazolyl carbocyanine iodide.

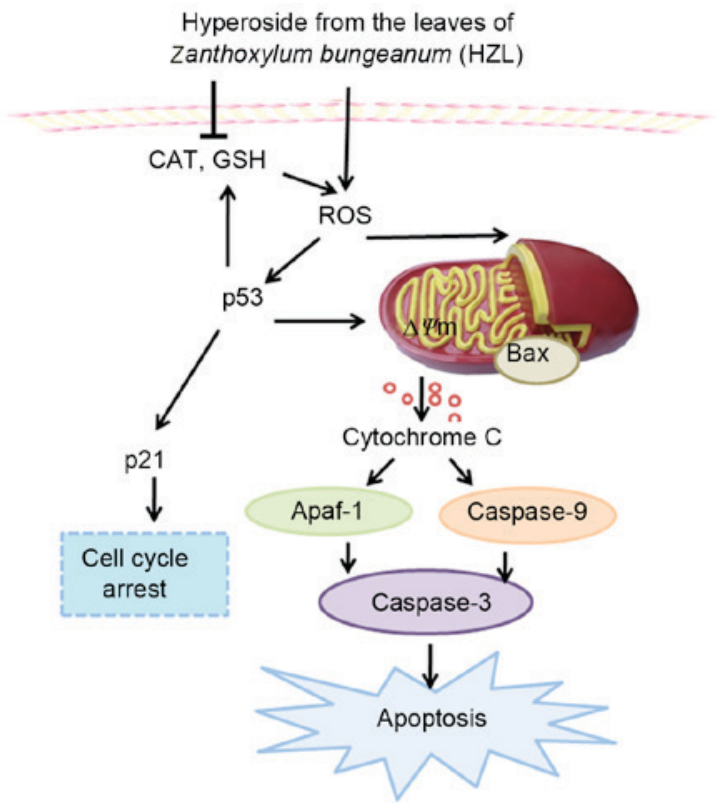

Figure 5. Suggested mechanism for HZL-induced cell cycle arrest and apoptosis in SW620 cells. Bax, Bcl-2-associated X protein; Apaf-1, apoptotic protease activating factor 1 ; CAT, catalase; ROS, reactive oxygen species; $\mathrm{GSH}$, glutathione peroxidase.

study detected upregulation in the protein expression levels of p53 and p21, suggesting that the upregulation of p53 and p21 was involved in HZL-induced G2/M cell-cycle arrest.

Apoptosis is a regulated programmed cell death process, which provides an effective non-inflammatory mechanism for the removal of redundant or damaged cells from tissues, thereby attaining tissue homeostasis (35). Tumor suppressor p53 is also an important activator of the intrinsic apoptotic pathway. The upregulation of p53 is commonly observed following exposure to DNA-damaging agents, including oxidative stress or UV light (36). Tumor suppressor p53 has been demonstrated to differentially regulate the levels of $\mathrm{Bcl}-2$ and $\mathrm{Bax}$ in vitro and in vivo (37). The increased production of Bax following p53 activation is due to p53 being a direct transactivator of the gene expression of Bax (37). The decreased expression of Bax in the present study was concomitant with the decreased expression of p53 and suggested that the apoptosis induced by HZL in SW620 cells may be controlled by hyperoside via the p53 pathway.

The collapse of $\Delta \Psi_{\mathrm{m}}$ is considered to coincide with the opening of mitochondrial permeability transition pores, leading to the release of cytochrome $c$ into the cytosol. In the cytoplasm, cytochrome $c$ combines with caspase-9, Apaf-1 and dATP to form the apoptosome complex, which in turn activates caspases-9, 3 and 7 (38). Caspase-9 and its cofactor, Apaf-1, are essential downstream components of p53 in Myc-induced apoptosis (39). In the present study, HZL upregulated the expression levels of cytochrome $c$, Apaf-1, caspase-9 and caspase-3, compared with the control group (Fig. 3). These findings suggested that p53 and the caspase-dependent signaling pathway were involved in HZL-induced apoptosis.

Reduced $\Delta \Psi_{\mathrm{m}}$ can lead to increased generation of ROS and apoptosis. In this context, Zeng et al (40) suggested that hyperoside alters the $\Delta \Psi_{\mathrm{m}}$ and induces mitochondrial collapse. Similar results were obtained in the present study when SW620 cells were treated with HZL. These findings, along with other those of others, suggested that hyperoside acts as an antiproliferative agent through the overproduction of ROS, induction of apoptosis and loss of $\Delta \Psi_{\mathrm{m}}$. The p53 protein has been suggested as a critical regulator of intracellular ROS levels. Upon activation following DNA damage, p53 can activate several genes, which results in increased 
ROS generation, contributing to the induction of apoptosis in cells with unrepaired DNA damage (41).

It has been found that human colorectal tumors (adenomas and carcinomas) have increased levels of different markers of oxidative stress, including increased levels of ROS, nitric oxide (NO) (42), lipid peroxides, GSH-Px and CAT (43). Alternative mechanisms for flavonoid pro-oxidant toxicity involve numerous peroxidases, which catalyze the oxidation of polyphenols. The intracellular phenoxyl radicals (redox-cycling phenols) formed by myeloperoxidase in neutrophils also induce lipid peroxidation and can co-oxidize GSH to form thiol radicals with concomitant oxygen release. The levels of CAT may be critical in cell-induced resistance to the effects of anticancer drugs, which upregulate p53 (44). Kang et al (45) demonstrated that p53 upregulates ROS generation by suppressing the activity of CAT in response to DNA damage. p53-mediated GSH depletion was found to enhance the cytotoxicity of NO in silibinin-treated human cervical carcinoma and HeLa cells (46). In the present study, HZL inhibited the mRNA expression levels of CAT and GSH-Px in a dose-dependent manner, suggesting that p53 was associated with the expression of genes encoding antioxidant enzymes.

The complex mechanism of HZL-induced cell cycle arrest and apoptosis in SW620 cells is demonstrated in Fig. 5. The results revealed that HZL increased the accumulation of ROS via inhibition of the gene expression of CAT and GSH, which resulted in cell cycle G2/M phase arrest and apoptosis. Further mechanistic investigations revealed that the induction of cell cycle $\mathrm{G} 2 / \mathrm{M}$ phase arrest and apoptosis was associated with the upregulated expression of p53, p21 and Bax in the SW620 cells. Consequently, the $\Delta \Psi_{\mathrm{m}}$ was reduced, which accelerated the release of cytochrome $c$ into the cytoplasm leading to apoptosis of the SW620 cells via the caspase-dependent pathway. These findings suggested that hyperoside may be a potent chemopreventive agent against human $\mathrm{CRC}$, acting through induction of the caspase-dependent apoptosis and p53 signaling pathways. Further investigations are warranted to determine the clinical efficacy and precise molecular mechanism by which hyperoside inhibits cancer cell growth.

\section{Acknowledgements}

The present study was financed by the Special Fund for Forest Scientific Research in Public Welfare (grant no. 201304811), the National Natural Science Foundation of China (grant no. 31101266) and the Scientific and Technical Foundation of Shaanxi Province (grant no. 2014JM4100). It was also partly supported by the Institute of Mitochondrial Biology and Medical, Xi'an Jiaotong University, Xi'an, China.

\section{References}

1. Amado NG, Predes D, Moreno MM, Carvalho IO, Mendes FA and Abreu JG: Flavonoids and Wnt/ $\beta$-catenin signaling: Potential role in colorectal cancer therapies. Int J Mol Sci 15: 12094-12106, 2014

2. Luo X, Yu X, Liu S, Deng Q, Liu X, Peng S, Li H, Liu J and Cao Y: The role of targeting kinase activity by natural products in cancer chemoprevention and chemotherapy (Review) Oncol Rep 34: 547-554, 2015.
3. Miura K, Satoh M, Kinouchi M, Yamamoto K, Hasegawa Y, Kakugawa Y, Kawai M, Uchimi K, Aizawa H, Ohnuma S, et al: The use of natural products in colorectal cancer drug discovery. Expert Opin Drug Discov 10: 411-426, 2015.

4. Xiong QB and Shi DW: Morphological and histological studies of Chinese traditional drug 'hua jiao' (Pericarpium Zanthoxyli) and its allied drugs. Yao Xue Xue Bao 26: 938-947, 1991 (In Chinese).

5. Zhang Y, Luo Z and Wang D: Efficient quantification of the phenolic profiles of Zanthoxylum bungeanum leaves and correlation between chromatographic fingerprint and antioxidant activity. Nat Prod Res 29: 2024-2029, 2015.

6. Zhang Y, Wang D, Yang L, Zhou D and Zhang J: Purification and characterization of flavonoids from the leaves of Zanthoxylum bungeanum and correlation between their structure and antioxidant activity. PLoS One 9: e105725, 2014.

7. Yang LC, Li R, Tan J and Jiang ZT: Polyphenolics composition of the leaves of Zanthoxylum bungeanum Maxim. Grown in Hebei, China, and their radical scavenging activities. J Agric Food Chem 61: 1772-1778, 2013.

8. Zhang Y,LuoZ, Wang D, He F and Li D: Phytochemical profiles and antioxidant and antimicrobial activities of the leaves of Zanthoxylum bungeanum. ScientificWorldJournal 2014: 181072,2014

9. Yang Q, Cao W, Zhou X, Cao W, Xie Y and Wang S: Anti-thrombotic effects of $\alpha$-linolenic acid isolated from Zanthoxylum bungeanum Maxim seeds. BMC Complement Altern Med 14: 348, 2014

10. Wu T, Zhong L, Hong Z, Li Y, Liu X, Pan L, Xin H and Zhu Y: The effects of Zanthoxylum bungeanum extract on lipid metabolism induced by sterols. J Pharmacol Sci 127: 251-259, 2015.

11. Sukito A and Tachibana S: Isolation of hyperoside and isoquercitrin from Camellia sasanqua as antioxidant agents. Pak J Biol Sci 17: 999-1006, 2014.

12. Yang FQ, Liu M, Li W, Che JP, Wang GC and Zheng JH: Combination of quercetin and hyperoside inhibits prostate cancer cell growth and metastasis via regulation of microRNA21. Mol Med Rep 11: 1085-1092, 2015.

13. Huo Y, Yi B, Chen M, Wang N, Chen P, Guo C and Sun J: Induction of Nur77 by hyperoside inhibits vascular smooth muscle cell proliferation and neointimal formation. Biochem Pharmacol 92: 590-598, 2014.

14. Li FR, Yu FX, Yao ST, Si YH, Zhang W and Gao LL: Hyperin extracted from Manchurian rhododendron leaf induces apoptosis in human endometrial cancer cells through a mitochondrial pathway. Asian Pac J Cancer Prev 13: 3653-3656, 2012.

15. Lee JH, Ahn J, Kim JW, Lee SG and Kim HP: Flavonoids from the aerial parts of Houttuynia cordata attenuate lung inflammation in mice. Arch Pharm Res 38: 1304-1311, 2015.

16. Fu T, Wang L, Jin XN, Sui HJ, Liu Z and Jin Y: Hyperoside induces both autophagy and apoptosis in non-small cell lung cancer cells in vitro. Acta Pharmacol Sin 37: 505-518, 2016.

17. Li W, Liu M, Xu YF, Feng Y, Che JP, Wang GC and Zheng JH: Combination of quercetin and hyperoside has anticancer effects on renal cancer cells through inhibition of oncogenic microRNA-27a. Oncol Rep 31: 117-124, 2014.

18. Khan HY, Zubair H, Ullah MF, Ahmad A and Hadi SM: A prooxidant mechanism for the anticancer and chemopreventive properties of plant polyphenols. Curr Drug Targets 13: 1738-1749, 2012

19. Bayir H, Fadeel B, Palladino MJ, Witasp E, Kurnikov IV, Tyurina YY, Tyurin VA, Amoscato AA, Jiang J, Kochanek PM, et al: Apoptotic interactions of cytochrome $c$ : Redox flirting with anionic phospholipids within and outside of mitochondria. Biochim Biophys Acta 1757: 648-659, 2006.

20. Li PF, Dietz R and von Harsdorf R: p53 regulates mitochondrial membrane potential through reactive oxygen species and induces cytochrome c-independent apoptosis blocked by Bcl-2. EMBO J 18: 6027-6036, 1999.

21. The Ninth Chinese Pharmacopoeia, Commission of the People's Republic of China. Pharmacopoeia of the People's Republic of China. The Medicine Science and Technology Press of China, 2010.

22. Livak KJ and Schmittgen TD: Analysis of relative gene expression data using real-time quantitative PCR and the 2(-Delta Delta C(T)) method. Methods 25: 402-408, 2001.

23. Procházková $\mathrm{D}$, Bousová I and Wilhelmová N: Antioxidant and prooxidant properties of flavonoids. Fitoterapia 82: 513-523, 2011. 
24. Catanzaro D, Ragazzi E, Vianello C, Caparrotta L and Montopoli M: Effect of quercetin on cell cycle and cyclin expression in ovarian carcinoma and osteosarcoma cell lines. Nat Prod Commun 10: 1365-1368, 2015.

25. Celano M, Maggisano V, De Rose RF, Bulotta S, Maiuolo J, Navarra M and Russo D: Flavonoid fraction of citrus reticulata juice reduces proliferation and migration of anaplastic thyroid carcinoma cells. Nutr Cancer 67: 1183-1190, 2015

26. Song H, Bao J, Wei Y, Chen Y, Mao X, Li J, Yang Z and Xue Y: Kaempferol inhibits gastric cancer tumor growth: An in vitro and in vivo study. Oncol Rep 33: 868-874, 2015.

27. Li C, Yang X, Chen C, Cai S and Hu J: Isorhamnetin suppresses colon cancer cell growth through the PI3K-Akt-mTOR pathway. Mol Med Rep 9: 935-940, 2014.

28. Zhang N, Ying MD, Wu YP, Zhou ZH, Ye ZM, Li H and Lin DS: Hyperoside, a flavonoid compound, inhibits proliferation and stimulates osteogenic differentiation of human osteosarcoma cells. PLoS One 9: e98973, 2014.

29. Lu M, Huang W, Bao N, Zhou G and Zhao J: The flavonoid ampelopsin inhibited cell growth and induced apoptosis and G0/G1 arrest in human osteosarcoma MG-63 cells in vitro. Pharmazie 70: 388-393, 2015.

30. Yi JL, Shi S, Shen YL, Wang L, Chen HY, Zhu J and Ding Y: Myricetin and methyl eugenol combination enhances the anticancer activity, cell cycle arrest and apoptosis induction of cis-platin against HeLa cervical cancer cell lines. Int J Clin Exp Pathol 8: 1116-1127, 2015.

31. Wang P, Wang B, Chung S, Wu Y, Henning SM and Vadgama JV: Increased chemopreventive effect by combining arctigenin, green tea polyphenol and curcumin in prostate and breast cancer cells. RSC Adv 4: 35242-35250, 2014.

32. Wu Z, Wu L, Li L, Tashiro S, Onodera S and Ikejima T: p53-mediated cell cycle arrest and apoptosis induced by shikonin via a caspase-9-dependent mechanism in human malignant melanoma A375-S2 cells. J Pharmacol Sci 94: 166-176, 2004.

33. Zhu F, Dollé ME, Berton TR, Kuiper RV, Capps C, Espejo A, McArthur MJ, Bedford MT, van Steeg H, de Vries A and Johnson DG: Mouse models for the p53 R72P polymorphism mimic human phenotypes. Cancer Res 70: 5851-5859, 2010.

34. Waga S, Hannon GJ, Beach D and Stillman B: The p21 inhibitor of cyclin-dependent kinases controls DNA replication by interaction with PCNA. Nature 369: 574-578, 1994.
35. Fiers W, Beyaert R, Declercq W and Vandenabeele P: More than one way to die: Apoptosis, necrosis and reactive oxygen damage. Oncogene 18: 7719-7730, 1999.

36. Pallardy M, Perrin-Wolff $M$ and Biola A: Cellular stress and apoptosis. Toxicol In Vitro 11: 573-578, 1997.

37. Miyashita T, Krajewski S, Krajewska M, Wang HG, Lin HK, Liebermann DA, Hoffman B and Reed JC: Tumor suppressor p53 is a regulator of bcl-2 and bax gene expression in vitro and in vivo. Oncogene 9: 1799-1805, 1994.

38. Ni CH, Yu CS, Lu HF, Yang JS, Huang HY, Chen PY, Wu SH, Ip SW, Chiang SY, Lin JG and Chung JG: Chrysophanol-induced cell death (necrosis) in human lung cancer A549 cells is mediated through increasing reactive oxygen species and decreasing the level of mitochondrial membrane potential. Environ Toxicol 29: 740-749, 2014.

39. Soengas MS, Alarcón RM, Yoshida H, Giaccia AJ, Hakem R, Mak TW and Lowe SW: Apaf-1 and caspase-9 in p53-dependent apoptosis and tumor inhibition. Science 284: 156-159, 1999.

40. Zeng KW, Wang XM, Ko H, Kwon HC, Cha JW and Yang HO: Hyperoside protects primary rat cortical neurons from neurotoxicity induced by amyloid $\beta$-protein via the $\mathrm{PI} 3 \mathrm{~K} / \mathrm{Akt} / \mathrm{Bad} / \mathrm{Bcl}(\mathrm{XL})$-regulated mitochondrial apoptotic pathway. Eur J Pharmacol 672: 45-55, 2011

41. Liu S, Yan B, Lai W, Chen L, Xiao D, Xi S, Jiang Y, Dong X, An J, Chen X, et al: As a novel p53 direct target, bidirectional gene $\mathrm{HspB} 2 / \alpha \mathrm{B}$-crystallin regulates the ROS level and Warburg effect. Biochim Biophys Acta 1839: 592-603, 2014.

42. Haklar G, Sayin-Ozveri E, Yüksel M, Aktan AO and Yalcin AS: Different kinds of reactive oxygen and nitrogen species were detected in colon and breast tumors. Cancer Lett 165: 219-224, 2001

43. Rainis T, Maor I, Lanir A, Shnizer S and Lavy A: Enhanced oxidative stress and leucocyte activation in neoplastic tissues of the colon. Dig Dis Sci 52: 526-530, 2007.

44. Bai J and Cederbaum AI: Catalase protects HepG2 cells from apoptosis induced by DNA-damaging agents by accelerating the degradation of p53. J Biol Chem 278: 4660-4667, 2003.

45. Kang MY, Kim HB, Piao C, Lee KH, Hyun JW, Chang IY and You HJ: The critical role of catalase in prooxidant and antioxidant function of p53. Cell Death Differ 20: 117-129, 2013.

46. Fan S, Yu Y, Qi M, Sun Z, Li L, Yao G, Tashiro S, Onodera S and Ikejima T: P53-mediated GSH depletion enhanced the cytotoxicity of NO in silibinin-treated human cervical carcinoma HeLa cells. Free Radic Res 46: 1082-1092, 2012. 